\title{
Integrated human/SARS-CoV-2 metabolic models present novel treatment strategies against COVID-19
}

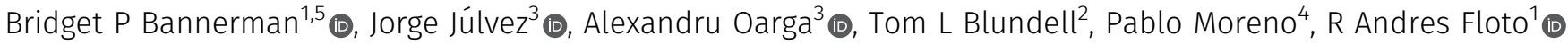

\begin{abstract}
The coronavirus disease 2019 (COVID-19) pandemic caused by the new coronavirus (SARS-COV-2) is currently responsible for more than 3 million deaths in 219 countries across the world and with more than 140 million cases. The absence of FDA-approved drugs against SARS-CoV-2 has highlighted an urgent need to design new drugs. We developed an integrated model of the human cell and SARS-CoV-2 to provide insight into the virus' pathogenic mechanism and support current therapeutic strategies. We show the biochemical reactions required for the growth and general maintenance of the human cell, first, in its healthy state. We then demonstrate how the entry of SARS-CoV- 2 into the human cell causes biochemical and structural changes, leading to a change of cell functions or cell death. A new computational method that predicts 20 unique reactions as drug targets from our models and provides a platform for future studies on viral entry inhibition, immune regulation, and drug optimisation strategies. The model is available in BioModels (https://www.ebi.ac.uk/biomodels/MODEL2007210001) and the software tool, findCPcli, that implements the computational method is available at https://github.com/findCP/findCPcli.
\end{abstract}

DOI 10.26508/lsa.202000954 | Received 10 November 2020 | Revised 26 July 2021 | Accepted 27 July 2021 | Published online 5 August 2021

\section{Introduction}

SARS-COV-2, the causative agent of COVID-19, belongs to a group of viruses commonly known as $\beta$-coronavirus. This class of viruses is responsible for mild-to-fatal respiratory tract infections in animals and birds. Whereas the common cold is more commonly associated with the mild forms of the disease, the previous MERS and SARS-2002 infections and, currently, COVID-19 belong to the group of fatal diseases. The genome of the virus responsible for the ongoing COVID-19 disease, SARS-CoV-2, has $~ 80 \%$ sequence identity to SARS-CoV and is $96 \%$ identical at the whole-genome level to a bat coronavirus (Zhou et al, 2020). SARS-CoV-2 affects the lower respiratory tract cells and the upper cells in the pharyngeal region (Chen et al, 2020; Huang et al, 2020), and the viral infections range from asymptomatic, mild, moderate, and severe cases. Previous studies in China show that $86 \%$ of cases of infection and the contagiousness of the virus were undocumented before travel restrictions were imposed (Li et al, 2020). In addition, the interim results from the Solidarity international clinical trial conducted by the World Health Organization confirmed that only corticosteroids are effective against severe and critical cases of COVID-19. The report shows little or no benefit from the other four treatments evaluated (remdesivir, hydroxychloroquine, lopinavir/ritonavir, and interferon) on overall mortality against COVID-19 (Dyer, 2020; Pan et al, 2021). Therefore, there are still many factors to unravel regarding the stages of infection and transmissibility patterns of the virus to achieve good treatment management strategies. Studies in France demonstrate the transmission potential of asymptomatic persons and suggest varying dynamics of transmission in children (Danis et al, 2020). The human angiotensinconverting enzyme 2 (human-ACE-2 protein) has been identified as the cell receptor for both the SARS-2002 virus and SARS-CoV-2. The ACE-2 enzyme, which has the primary function of controlling blood pressure, is usually found in the epithelial cells of the heart, lungs, kidneys, and intestine (Donoghue et al, 2000; Hamming et al, 2004; Liu et al, 2020).

The mechanism of replication of SARS-CoV-2 in the human cell involves an initial binding and attachment of the spike (S) glycoprotein to the angiotensin-converting enzyme 2 (ACE2) receptor of its host. During endocytosis, the virus's genetic material is injected into the host cell, where it loses its protective envelope (Fig 1). The subsequent assembly and maturation of viral proteins lead to cell death and proliferation of the virus within the human body.

The lack of FDA-approved drugs against COVID-19, coupled with the difficulties encountered globally in containing the virus, prompted the WHO to declare the outbreak a pandemic in March 2020. This has led to intensified efforts around the world to fight this disease. Previous studies in drug target identification against viral diseases such as Zika, chikungunya, and dengue by Aller et al, (2018) introduced a system of integrating the host's macrophage and viral metabolic networks to predict a set of host reactions which, when constrained, can inhibit viral production. A recent study by Renz et al, (2020) demonstrates a similar approach and predicts drug targets against SARS-CoV-2. Targets of known antiviral drugs predicted from both studies, using a macrophage metabolic model (Bordbar et al, 2010) demonstrate the applicability of the integrated human/virus metabolic modelling in drug target identification. The alveolar macrophage host model (Bordbar et al, 2010) used to

\footnotetext{
${ }^{1}$ Molecular Immunity Unit, Department of Medicine, University of Cambridge, Cambridge, UK ${ }^{2}$ Department of Biochemistry, University of Cambridge, Cambridge, UK ${ }^{3}$ Department of Computer Science and Systems Engineering, University of Zaragoza, Zaragoza, Spain ${ }^{4}$ EMBL-EBI, European Bioinformatics Institute, Hinxton, UK ${ }^{5}$ The Center for Research and Interdisciplinarity, Paris, France
}

Correspondence: bpc28@cam.ac.uk; arf27@cam.ac.uk 


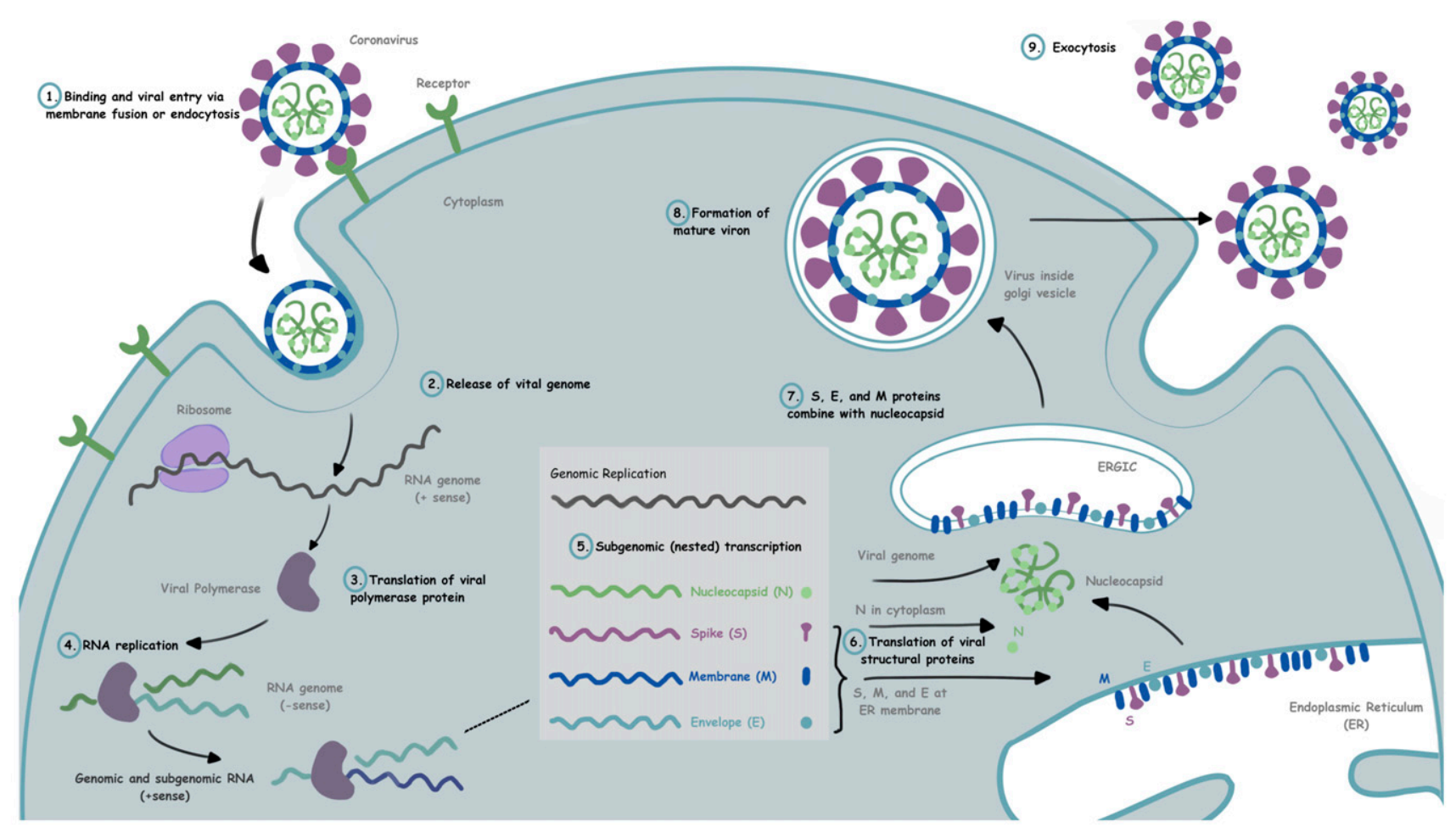

Adapted from "Coronavirus Replication Cycle", by BioRender.com (2020). Retrieved from https://app.biorender.com/biorender-templates

Figure 1. The mechanism of replication of SARS-CoV-2 in the human cell.

illustrate the metabolic interactions between multiple organisms was constructed from the Homo sapiens Recon 1 model (Duarte et al, 2007) based on previously built algorithms. This was followed by a set of manual curation processes to construct the context-specific alveolar macrophage host model (Bordbar et al, 2010). Similarly, Wang et al (2012) developed several draft context-specific models using the Human Recon 1 model.

We have built on these approaches by developing an integrated epithelial cell/SARS-CoV-2 metabolic model and used a combination of structural and dynamical analyses to assess the model and make predictions. We have applied the recently released community standards to facilitate our development of a standardised model for the systems biology international community and used the MEMOTE quality control software to assess and compare our model with previously developed GEMS (Carey et al, 2020, Lieven et al, 2020). We have designed a novel computational method and developed a software tool (findCPCli) to carry out such analyses and to predict drug targets. Both the model and the tool have been uploaded to public repositories (see the Data Availability section). We have also performed a comparative analysis of our model and other previously generated cell-type models.

\section{Results}

\section{Construction of the human airway epithelial cell and the SARS-CoV-2 virus}

We constructed an integrated genome-scale metabolic model (GEM) of the human airway epithelial cell with the SARS-CoV-2 virus using the methods described in Aller et al (2018) and Renz et al (2020). We used a previous draft reconstruction of the epithelial cell (Wang et al, 2012) and improved on the context-specificity of the GEM with the addition and simulation of data from the gene expression omnibus on COBRApy (Ebrahim et al, 2013). We performed further refinement from literature and by using the human metabolic networks in the HumanCyc database (Trupp et al, 2010) and Recon3D (Brunk et al, 2018). Model checks were done with MEMOTE, and refinements were performed with MetaNetX 4.2, BiGG, ChEBI, MetaCyc, and PubChem databases (Caspi et al, 2014; Hastings et al, 2016; Norsigian et al, 2020; Kim et al, 2021; Moretti et al, 2021). The new GEM contains 4,660 genes, 3,614 reactions, and 4,052 metabolites and conforms to the minimum standardised content for a newly published GEM based on recently published community standards (Carey et al, 2020); 100\% of the metabolites in (iHsaEC21) have a human-readable descriptive name, 100\% have an inchi key, $100 \%$ of metabolite annotation conformity with the BiGG database and in MetaNetX, Kyoto Encyclopedia of Genes and Genomes (KEGG), ChEBI, ModelSEED, HMDb, or MetaCyc (Caspi et al, 2014; Hastings et al, 2016; Wishart et al, 2018; Norsigian et al, 2020; Kanehisa et al, 2021; Kim et al, 2021; Moretti et al, 2021; Seaver et al, 2021); $100 \%$ of the metabolites have a charge and chemical formula with a charge balance of $75.3 \%$ (Supplemental Data 1). In addition, $97 \%$ of the reactions have a human-readable descriptive name, $100 \%$ of reactions conform with the BiGG database and as well as in MetaNetX, KEGG, ChEBI, ModelSEED, HMDb, or MetaCyc. The Gene IDs in the model are consistent with gene annotations in Uniprot with 92.5\% of reactions having Gene-Protein-Reaction associations and 71.9\% having the Enzyme Classification code. 


\section{Comparative analysis of integrated models of infected human epithelial cell and the macrophage cell with SARS-CoV-2}

For our study, we performed a comparative analysis of the essential and unique reactions needed for the viability of the virus in the epithelial cell/SARS-COV-2 integrated model and the GEM constructed by Renz et al (2020). Our results show how the virus heightens its virulence mechanisms by modifying the host's defences within different cell compartments. Consequently, we suggest treatment regimens based on different stages of viral infection and replication.

\section{Host-dependent metabolic pathways}

We initially demonstrated the biochemical requirements for the maintenance of the human airway epithelial and macrophage cells and used the integrated models to show the essential host reactions needed for the survival and viability of SARS-CoV-2 within the host's cell compartments. We have mapped the experimentally characterized human/SARS-CoV-2 protein-protein interaction data from Gordon et al (2020) on the in silico virus-integrated human macrophage and epithelial cells. Of the 334 metabolic pathways in the human metabolic network, 48 pathways including the biosynthesis and degradation pathways of amino acids, fatty acids, carbohydrates, amines, cofactors, and core components of the central mRNA metabolism are hijacked by the virus for its survival strategies (Fig 2).

The 48 metabolic pathways that were mapped to the proteinprotein interaction network produced by Gordon et al (2020) are referred to as PPi-pathway intersection nodes in this article (Table 1).
These include cysteine, methionine, and selenocysteine amino acid biosynthetic pathways; C20 prostanoid hormone biosynthetic pathways; and vitamin D3 and vitamin K epoxide cycle. The degradation pathways identified include the lysine, tryptophan, methionine, fatty acid degradation, ceramide, and sphingolipid recycling pathways; phospholipases degradation; and amine and heme degradation (Table 1).

Our results identify host dependency factors required for the SARS-CoV-2 virus infection, replication, survival, and viability within different cell compartments and provide insight into novel treatment strategies.

\section{Essential reactions for the host and viral metabolism}

The Flux Balance Analysis (FBA) method (Orth et al, 2010) was used to compute both the biomass maintenance of the cell in the absence of virus and the maximum growth rate of the virus in the cell (host optimum and virus optimum conditions). We identified 52 essential reactions in the macrophage (iAB-AM $\varnothing-1410)$ model and 10 reactions in the epithelial cell model (iHsaEC21) essential for the virus to propagate (Tables S1 and S2). It was also demonstrated that: (i) the maximal biomass maintenance of the macrophage cell in the absence of virus was 0.0269 (Table S1) and $0.012 \mathrm{~h}^{-1}$ for the human airway epithelial cell (Table S2); (ii) the maximum growth rate of the virus in the macrophage cell was 0.0144 and $0.0181 \mathrm{~h}^{-1}$ in the human airway epithelial cell. These numerical results mean that $0.0144 \mathrm{~h}^{-1}$ is the theoretical maximum of the growth rate of the virus in the human macrophage cell. If this flux is assigned to the viral growth

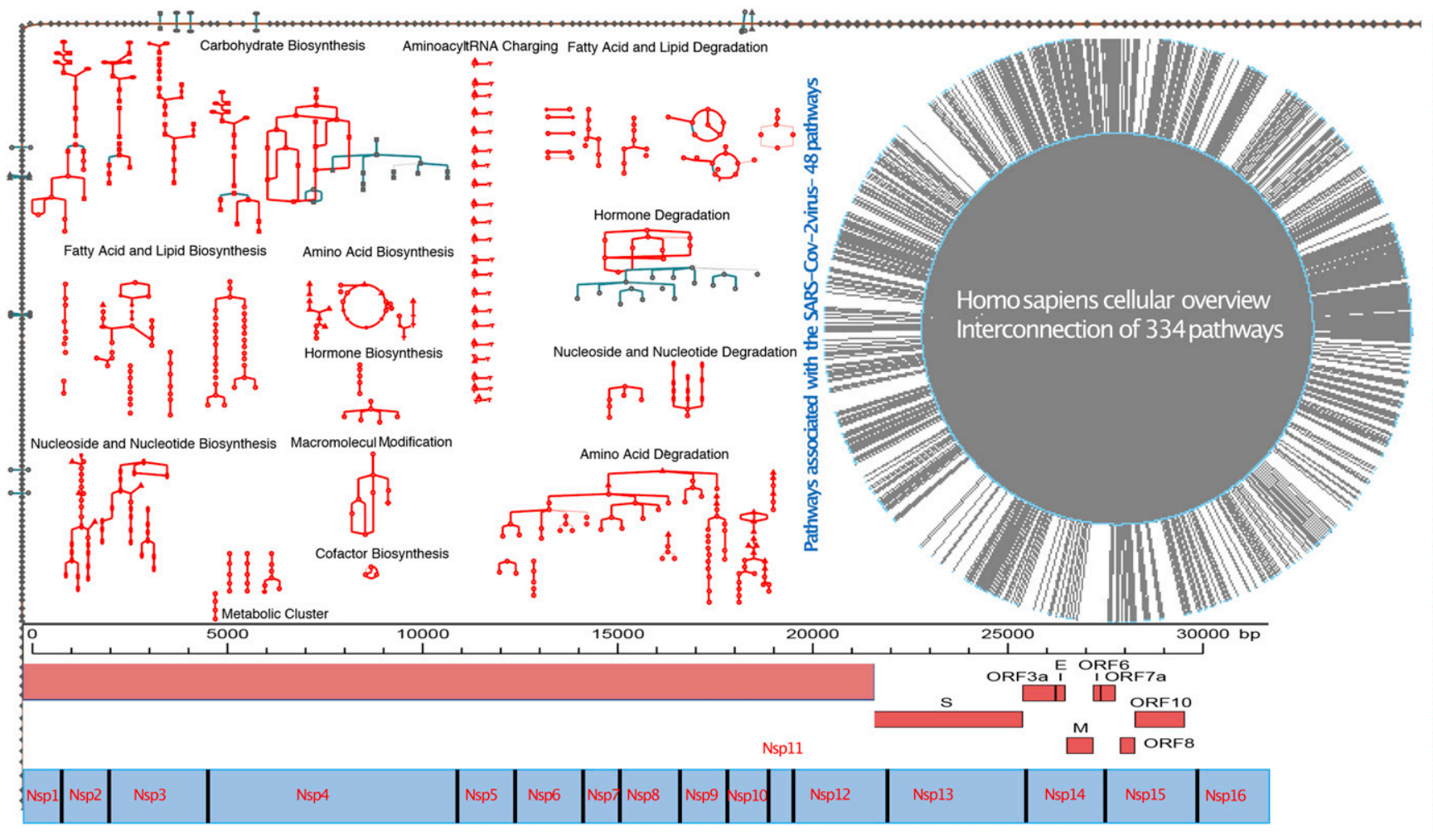

Figure 2. SARS-CoV-2 viral genome and host-dependent metabolic pathways. 
Table 1. List of bottleneck and essential enzymes on the PPi-pathway intersection nodes.

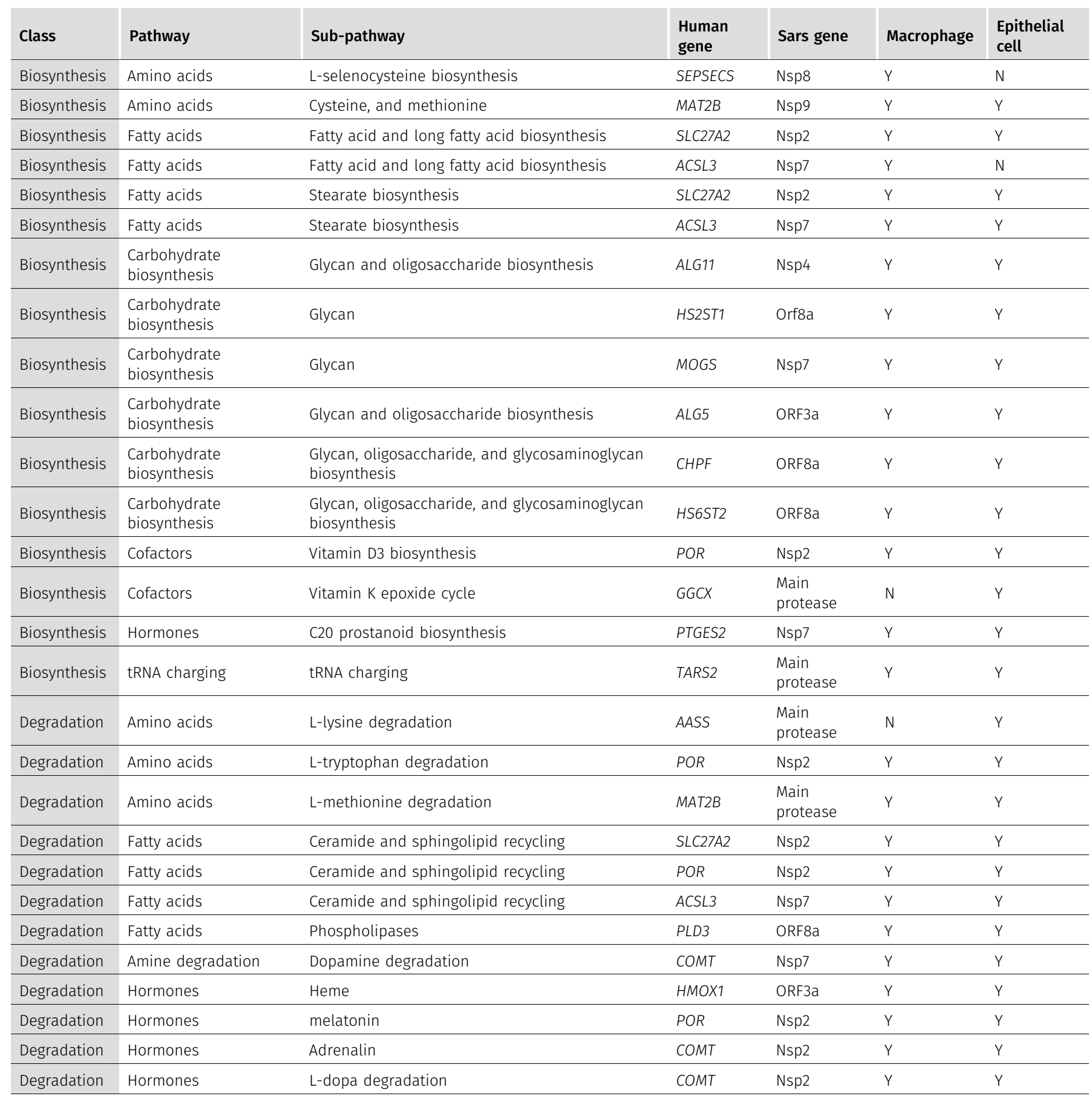

reaction, then flux variability analysis (FVA) (Orth et al, 2010) can be used to calculate the ranges of fluxes allowed for the remaining reactions in the cell while the virus is being replicated at its optimum condition. The execution of FVA under such conditions produced zero biomass maintenance of the host cell, that is, both the lower and upper flux bounds of the reaction indicate that the growth is zero. This means that if the virus is replicating at its maximum rate, then the cell will not be viable.

\section{Bottleneck reactions and the prioritization of potential drug targets}

The bottleneck reactions identified by the findCPCli tool are unique reactions of the metabolic network required for the growth and survival of the organism and, like chokepoint reactions, are potential drug targets (Yeh et al, 2004; Oarga et al, 2020). Although classical chokepoint reactions identify reactions that are the only 
producers or the only consumers of a given metabolite and consider just the model structure, we improve on this approach by using both the structural and dynamical information. FVA is used to compute flux bounds of the reactions, and in turn, to determine whether a given reaction is reversible or not. Reversibility will be used to obtain the sets of metabolites that can be produced and consumed by the reactions, and thus, to compute flux-dependent bottleneck reactions. This approach has been applied to the integrated Human/SARS-CoV-2 metabolic model within the airway epithelial cell and the macrophage cell to predict potential drug targets against SARS-CoV-2.

We initially identified 1,595 bottleneck reactions required for the virus' maintenance and replication in the human macrophage cell; these include pathways in lipid metabolism, coenzyme transport and metabolism, energy production and conversion, and amino acid and nucleotide transport and metabolism (Table S3). In the human airway epithelial cell, 1,598 bottleneck reactions were initially identified; these include the biosynthesis and degradation pathways of amino acids, fatty acids, carbohydrates, amines, cofactors, and some components of the central mRNA metabolism (Table S4).

Because each bottleneck reaction should be balanced by at least one other reaction that produces or consumes that metabolite, we have excluded reactions in the model with dead-end metabolites. The bottleneck reactions are potential drug targets as they are indispensable for the maintenance and replication of the virus within the host. To rank the potential drug targets identified, we prioritised enzymes for unique reactions that occur at the nodes of intersection between the bottleneck and essential reactions and the results from the human/virus protein-protein interaction network (Gordon et al, 2020) (Table 1). The following steps were taken to label the reactions which occur at the nodes of intersections between the referenced points above: (1) we initially identified both the bottleneck and essential reactions to the virus within the model, (2) we highlighted the interactions between the viral proteins and the human metabolic enzymes, and (3) we selected the reactions from the human/virus interactions which are also present in the list of bottleneck and/or essential reactions.

The enzymes of the reactions at the nodes of intersection are (1) host dependency factors identified by the model as necessary for the survival of the virus and (2) proteins from experimental datasets with high-confidence virus-human protein-protein interaction data; we refer to these points of intersection as PPi-pathway intersection nodes.

The PPi-pathway intersection (P-Pi) nodes identified are present in biosynthesis pathways such as the cysteine and S-adenosyl-Lmethionine biosynthetic pathways. In both pathways, the enzyme S-adenosylmethionine synthase (MAT2B) catalyses the phosphorylation reaction of methionine to S-adenosyl-L-methionine. During infection, the viral protein Nsp9 is seen to react with MAT2B (Gordon et al, 2020) (Fig 3A-C). Another viral protein, Nsp8, also interacts with the enzyme O-phosphoseryl-tRNA(Sec) selenium transferase (SEPSECS), which catalyses the last step of the L-selenocysteine biosynthesis pathway (Fig 4).

P-Pi nodes also occur in a network of various fatty acid and stearate biosynthetic pathways with Nsp2 interacting with the very long-chain acyl-COA synthetase (SLC27A2) (Fig 5A and B). The viral protein, Nsp2, also interacts with POR in other pathways including vitamin D3 biosynthesis (Fig 5C) and in L-tryptophan degradation, ceramide, and sphingolipid recycling pathways (Table 1).

In carbohydrate metabolism, a P-Pi node is identified at the glycan and oligosaccharide biosynthetic pathways, and specifically where two mannose residues are added in $\alpha(1 \rightarrow 2)$ linkages to the nascent oligosaccharide and catalysed by the enzyme ALG11. The viral protein Nsp4 interacts with ALG11 during the infection of the SARS-CoV-2 virus (Fig 6). Another viral protein, Nsp7, interacts with ACSL3 in the $y$-linolenate biosynthesis (Fig 7A). Nsp7 also reacts with ACSL3 and ORF8a interacts with HS2ST1 (Fig 7B), a key enzyme involved in the heparan sulfate biosynthesis pathway. The first enzyme of the $\mathrm{N}$-linked oligosaccharide processing pathway, mannosyloligosaccharide $\alpha$-1,2-glucosidase (MOGS), also interacts with Nsp7 and ORF8a (Fig 7C).

$\mathrm{P}$-Pi nodes specific to the human macrophage cell include the O-phosphoseryl-tRNA(Sec) selenium transferase in the L-selenocysteine biosynthetic pathway, which interacts with the viral protein Nsp8. The alkylglycerone-phosphate synthase/Nps7 P-Pi node, which is present in the phospholipid/plasmalogen biosynthetic pathway is also specific to the macrophage cell. Alternatively, PPi-pathway intersection nodes common to both human airway epithelial cell and the macrophage cell include the MAT2B/Nsp9 intersection pathways present in the cysteine metabolism and L-methionine degradation. P-Pi nodes specific to the human epithelial cell include the peptidyl-glutamate 4-carboxylase present in the Vitamin K epoxide cycle and the $\alpha$-aminoadipic semialdehyde synthase enzyme in the L-lysine degradation pathway.

\section{Discussion}

Metabolic pathway perturbations in the human cell due to COVID-19 reflect the viral entry and infection of SARS-CoV-2 and the immune regulation changes in the human body. We have used in silico models to study the interactions of SARS-CoV- 2 in the host and propose new treatment management regimens. We have built on studies using the human alveolar macrophage model iAB-AM $\varnothing-1410$ (Bordbar et al, 2010) as host cells and SARS-CoV-2 (Renz et al, 2020, 2021); influenza ((Aller et al 2018)), and tuberculosis as pathogens and developed a new integrated model of the human epithelial cell and SARS-CoV-2.

The initial draft epithelial cell model was based on the human Recon1 model and consists of 1,206 reactions, 994 metabolites, and 0 genes (Wang et al, 2012). Our revised iHsaEC21 epithelial cell model contains 4,660 genes, 3,614 reactions, and 4,052 metabolites initially scored $34 \%$ on MEMOTE (Lieven et al, 2020). After further refinements using Recon3D, HumanCyc, MetaNetX 4.2, BiGG, ChEBI, MetaCyc, and PubChem databases (Caspi et al, 2014; Hastings et al, 2016; Norsigian et al, 2020; Moretti et al, 2021) including manual curations with literature, $\mathrm{iHsaEC} 21$ now has a MEMOTE quality score of 51\% (Supplemental Data 1). Although the iAB-AM $\varnothing-1410$ (Bordbar et al, 2010) was also developed from the human Recon1 model, there was further refinement with literature, which attributed to its quality score of $45 \%$ (Supplemental Data 2) compared with the draft epithelial cell model from Wang et al (2012) which had a low-quality score of 19\% (Supplemental Data 3). 
(A) Methionine degradation

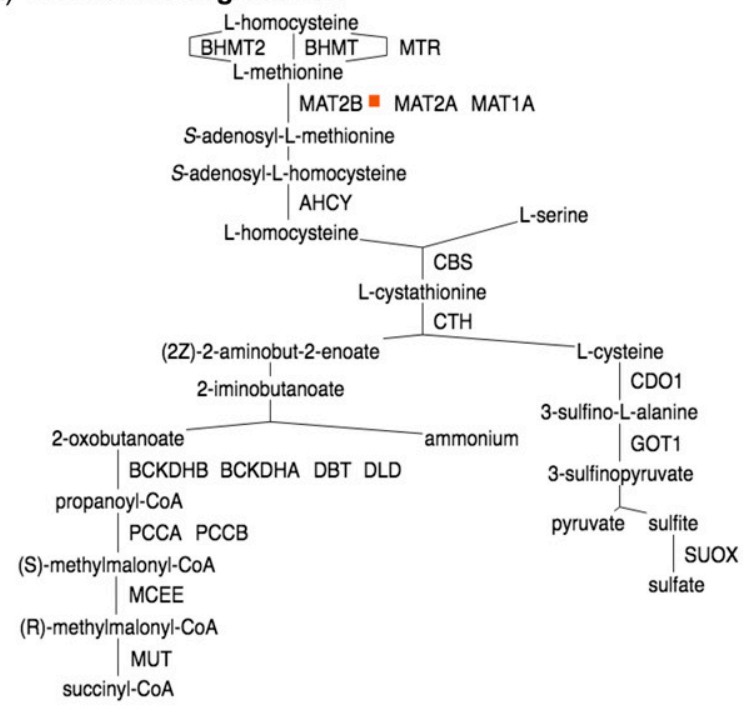

(C) Cysteine Biosynthesis

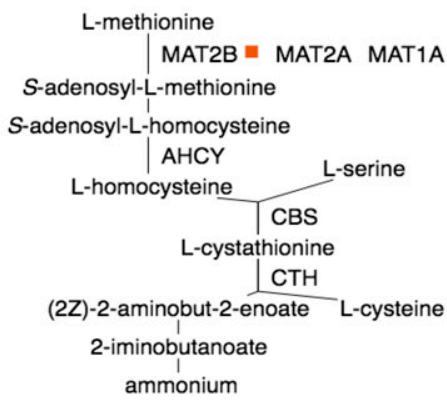

Figure 3. PPi-pathway intersection node-Nsp9.

(A) Methionine degradation. (B) Methionine salvage cycle. (C) Cysteine biosynthesis.
Previous studies have also demonstrated the role of the ACE2 as the receptor for both the SARS-COV and the SARS-COV-2. The ACE2 cells are expressed in the human airway epithelial cells (Hamming et al, 2004; Liu et al, 2020). In this study, we have constructed an integrated human epithelial cell and SARS-CoV-2 to provide insight into the infection patterns of the virus in the human body.

Our in silico comparative analyses of the SARS-CoV-2 viral infection between two different conditions (infected human macrophage and airway epithelial cells) show the requirements of viability of the virus between these two conditions. Our results complement previous efforts to propose drug targets and repurposing strategies including the SARS-CoV-2-Human Protein-Protein Interaction Map

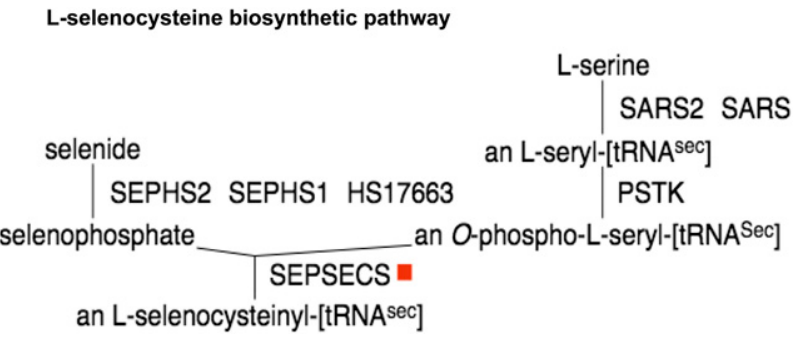

Figure 4. PPi-pathway intersection node-Nsp8.

\section{(B) Methionine salvage cycle}

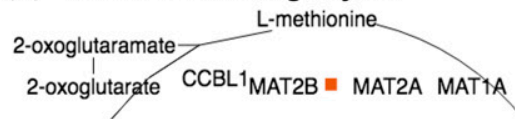$$
\text { ADI1 }
$$

1,2-dihydroxy-5-(methylsulfanyl)pent-1-en-3-one

ENOPH1

2-hydroxy-5-(methylsulfanyl)-
3-oxopent-1-enyl 1-phosphate

ENOPH1

5-(methylsulfanyl)-2,3-dioxopentyl 1-phosphate $S$-methyl-5-thio- $\alpha$-D-ribose 1-phosphate

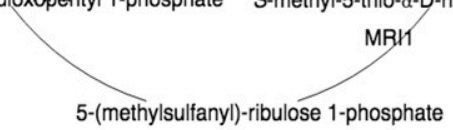

4-(methylsulfanyl)-2-oxobutanoate

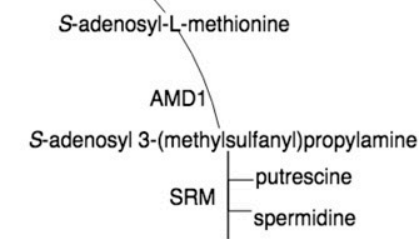

$S$-methyl-5'-thioadenosine MTAP

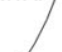

Gordon et al, (2020 and studies from Steward, 2020, which identified host dependency factors facilitating virus infection. We also provide additional resources to the COVID-19 disease map (Ostaszewski et al, 2020). In addition, we have designed a new algorithm (Oarga et al, 2020) and used the dynamic information of the human/virus models to predict new treatment regimens. Here, we demonstrate the flux changes from lipid metabolism, cofactor biosynthetic pathways, redox balance, and immune regulation indicative of pathogenic reactions arising from the COVID-19 viral infections.

\section{Inhibition of viral entry and replication}

The components of the plasma membrane, such as cholesterol and sphingolipid-rich lipid, Abu-Farha et al, (2020) are involved in virus penetration, entry, replication, and infection (Wang et al, 2008; AbuFarha et al, 2020). In this study, we demonstrate current therapeutic strategies that interfere with different stages of the viral cycle by targeting lipid metabolism and proposing new treatment strategies.

The dynamical changes of flux metabolism in our in-silico virus optimal models show a significant increase in viral infection. Four reactions involved in the biosynthesis of fatty acids with predicted non-zero fluxes in the host model exhibit an average increase of $190 \%$ in their maximum fluxes in the viral model (the maximum 
(A) Fatty acid oxidation

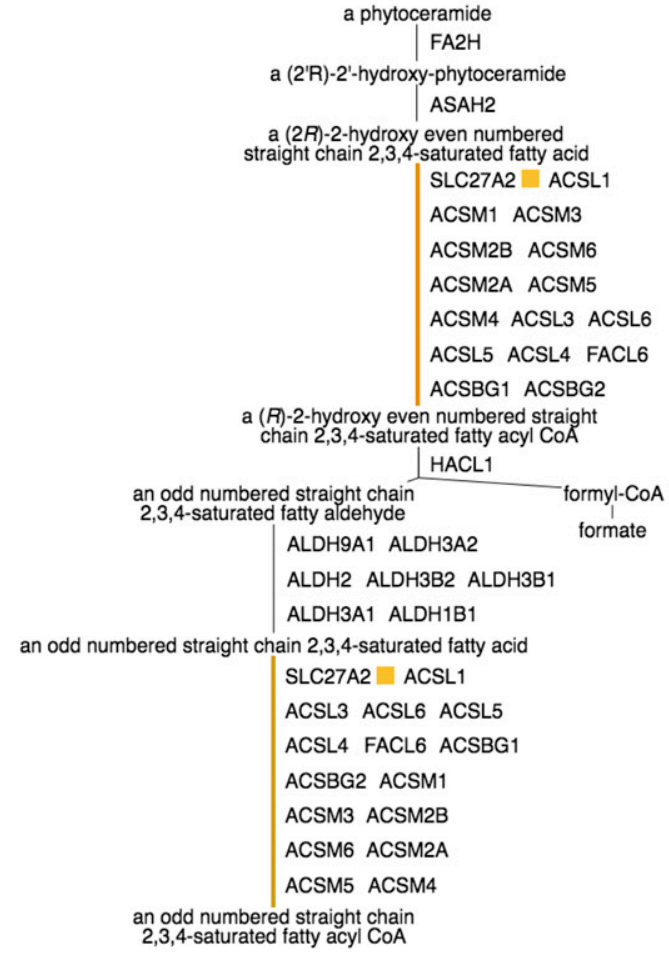

(B) Stearate Biosynthesis

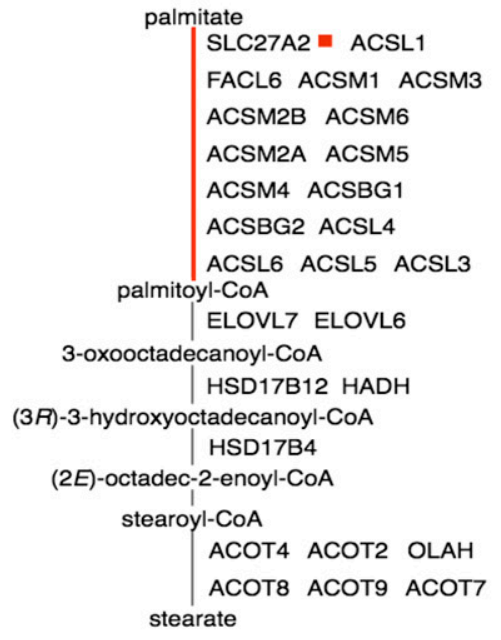

(C) Vitamin D3 Biosynthesis

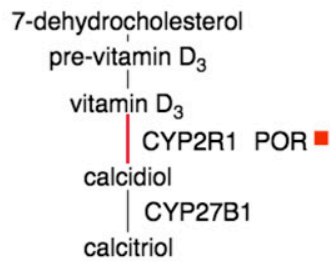

Figure 5. PPi-pathway intersection node-Nsp2.

(A) Fatty acid oxidation. (B) Stearate biosynthesis. (C) Vitamin D3 biosynthesis.

increase is 298\%). The average increase of 32 reactions in lipid metabolism with non-zero fluxes in the host model is $277 \%$ (the maximum increase is 498\%). Concerning sphingolipid metabolism, 14 of 15 reactions with non-zero fluxes in the host model exhibit an average increase of $228 \%$ (the maximum increase is 298\%) and similar increases in phospholipases and palmitic acid biosynthesis (Fig 5 A and B). We show an average increase of $190 \%$ in cholesterol and fatty acid metabolism during viral infection and demonstrate the essentiality of these pathways to SARS-CoV-2. Previous studies have shown that cholesterol and fatty acids are main components of the viral membranes and needed for viral replication (Heaton \& Randall, 2011); therefore, drugs inhibiting these pathways such as AM580, statins, and fibrate (Fiévet \& Staels, 2009) will be essential for both early and late stages of COVID-19.

Sphingolipids are composed of both hydrophobic and hydrophilic units and play a large role in the endocytic or exocytic viral entry processes into the cell (Dimitrov, 2004). The pH-dependent endocytic process is further enhanced by the presence of clathrin, a protein present in the plasma membrane, the Golgi apparatus and in the cytoplasm, whereas the exocytic route involves viral crossing through the plasma membrane at neutral $\mathrm{pH}$. Our results show a threefold increase in sphingolipid metabolism during viral infection (Fig 5); we hypothesise that drugs inhibiting sphingolipid metabolism and/or the endocytosis process will inhibit infection of SARS-CoV-2. Indeed, a sphingosine kinase-2 (SphK2) inhibitor, opaganib, which has proved beneficial in the treatment of COVID-19, is currently in global phase $2 / 3$ clinical trials and in US phase 2 studies. Previous studies also demonstrate that chloroquine and hydroxychloroquine elevate the $\mathrm{pH}$ of endosomes in the cells and directly inhibit endocytosis and the exocytic process (Munro et al, 1997; Devaux et al, 2020). More recent studies have also shown that artemisinin-inhibited endocytosis (Hoppe et al, 2004; Uzun \& Toptas, 2020). A recent study by Abu-Farha described other lipid-modifying drugs including LJ-001, arbidol, methyl-B-cyclodextrin (Blaising et al, 2013; de Wilde et al, 2014; Mazzon \& Marsh, 2019). We highlight critical reactions as drug targets for lipid metabolism for the SARS-CoV-2 virus (Table S2) and in our PPi-pathway intersection nodes (Table 1).

Our lists of bottleneck, essential reactions, and PPi-pathway intersection nodes also include critical points in the biosynthesis of phospholipids. We show that these reactions are essential for viral infection and replication (Table 1) and propose targeting the phospholipase enzyme or the interacting Nsp2 protein to inhibit viral replication. Our results support previous studies from Müller et al (2018) that targeting the phospholipase enzyme could inhibit the early stage of COVID-19.

\section{Redox homeostasis and antioxidant therapy}

Redox homeostasis refers to the ability of the cell to maintain its balance amidst infections and other unstable cellular environmental factors. Delgado-Roche and Mesta (2020) have described oxidative stress as a key player in severe acute respiratory syndrome coronavirus (SARS-CoV) infection with cytokine production. Foyer and Noctor (2005) have previously shown that antioxidants, such as glutathione and 


\section{$\mathrm{N}$-glycosylation initial phase}

a dolichyl phosphate UDP- $N$-acetyl- $\alpha$-D-glucosamine

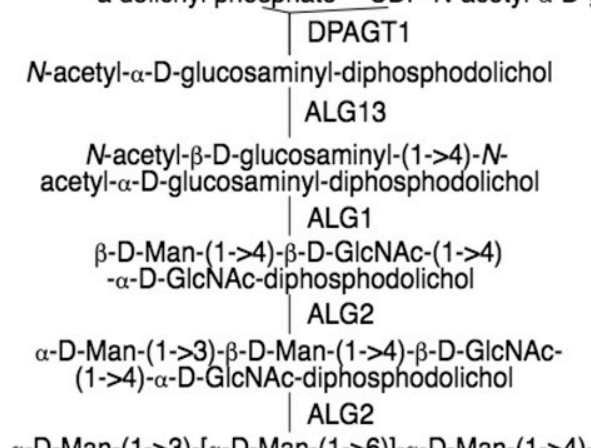

$\alpha$-D-Man-(1->3)-[ $\alpha$-D-Man-(1->6)]- $\alpha$-D-Man-(1->4)

$\beta$-D-GIcNAc-(1->4)- $\alpha$-D-GlcNAC-diphosphodolichol

| ALG11 1

$\alpha$-D-Man-(1->2)- $\alpha$-D-Man-(1->2)- $\alpha$-D-Man-

$(1->3)-[\alpha-D-M a n-(1->6)]-\beta-D-M a n-(1->4)-\beta-D$ -

GlcNAc-(1->4)- $\alpha$-D-GlcNAC-diphosphodolichol

$\alpha$-D-Man-(1->2)- $\alpha$-D-Man-(1->2)- $\alpha$-D-Man-

$\alpha-D-M a n-(1->2)-\alpha-D-M a n-(1->2)-\alpha-D-M a n-$
$(1->3)-[\alpha-D-M a n-(1->6)]-\beta-D-M a n-(1->4)-\beta-D-$

GlCNAC-(1->4)- $\alpha$-D-GICNAC-diphosphodolichol a dolichyl $\beta$-D-mannosyl phosphate

$$
\text { ALG3 }
$$

$\alpha$-D-Man-(1->2)- $\alpha$-D-Man-(1->2)- $\alpha$-D-Man-(1->3)-

$[\alpha$-D-Man-(1->3)- $\alpha$-D-Man- $(1->6)]-\beta$-D-Man-(1->4)

$\beta$-D-GIcNAc-(1->4)- $\alpha$-D-GICNAC-diphosphodolichol

$$
\text { I ALG9 }
$$

$\alpha$-D-Man-(1->2)- $\alpha$-D-Man-(1->2)- $\alpha$-D-Man-(1->3)-[ $\alpha$-D-

Man-(1->2)- $\alpha$-D-Man-(1->3)- $\alpha$-D-Man-(1->6)]- $\beta$-D-Man-

(1->4)- $\beta$-D-GlcNAc-(1->4)- $\alpha$-D-GlcNAc-diphosphodolichol

ALG12

$\alpha$-D-Man-a-(1->2)- $\alpha$-D-Man-(1->2)- $\alpha$-D-Man-

$(1->3)-[\alpha-D-M a n-(1->2)-\alpha-D-M a n-(1->3)-[\alpha-D$

Man- $(1->6)]-\alpha-D-M a n-(1->6)]-\beta-D-M a n-(1->4)-\beta-$

D-GIcNAC-(1->4)- $\alpha$-D-GlcNAC-diphosphodolichol I ALG9

$\alpha$-D-Man- $(1->2)-\alpha-D-M a n-(1->2)-\alpha-D-M a n-(1->3)$ [ $\alpha$-D-Man- $(1->2)-\alpha$-D-Man- $(1->3)-[\alpha-D-M a n-(1->2)-$ a dolichyl $\beta$-D-glucosyl phosphate $\alpha$-D-Man-(1->6)]- $\alpha$-D-Man- $(1->6)]-\beta$-D-Man-(1->4).

$\beta$-D-GIcNAC-(1->4)- $\alpha$-D-GIcNAC-diphosphodolichol a dolichyl $\beta$-D-glucosyl phosphate
Figure 6. PPi-pathway intersection node-Nsp4.

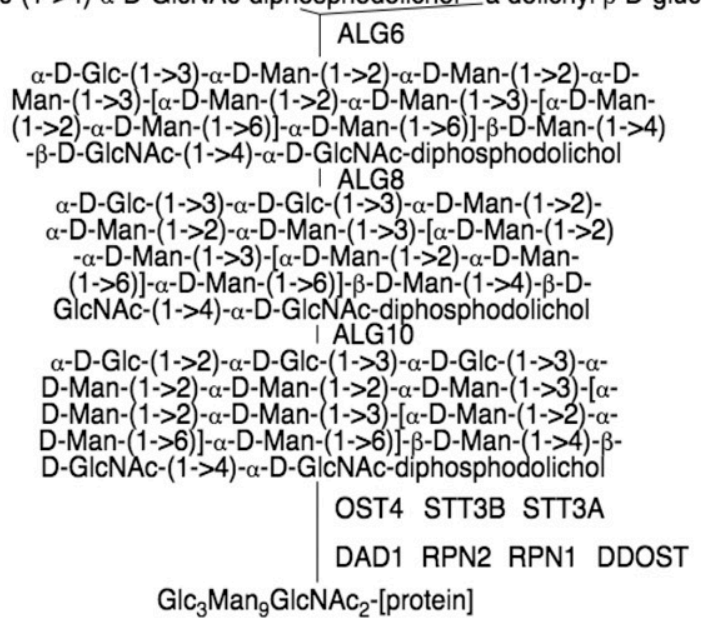

ascorbate, are important metabolites for the cellular redox state. Our studies have identified key target enzymes involved in the metabolism of glutathione and ascorbic acid as bottleneck and essential reactions, including glutathione synthase, glutathione peroxidase, and ascorbic acid oxidase (Table S2). We also demonstrate an increase in the flux of these enzymatic reactions on infection of the virus. In a recent study,
Horowitz et al (2020) previously demonstrated how the use of high dose oral and/or IV glutathione on severe outcomes of SARS-CoV-2 led to favourable treatment outcomes. Other studies have shown that steroids such as dexamethasone and Methylprednisolone are used to treat severe cases of COVID-19. Because of the possible side effects of steroid treatment, we propose the use of glutathione as therapy for severe cases 


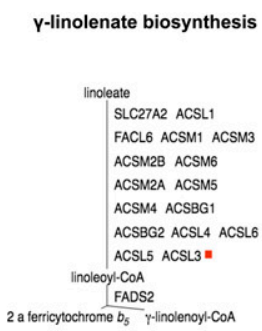

A

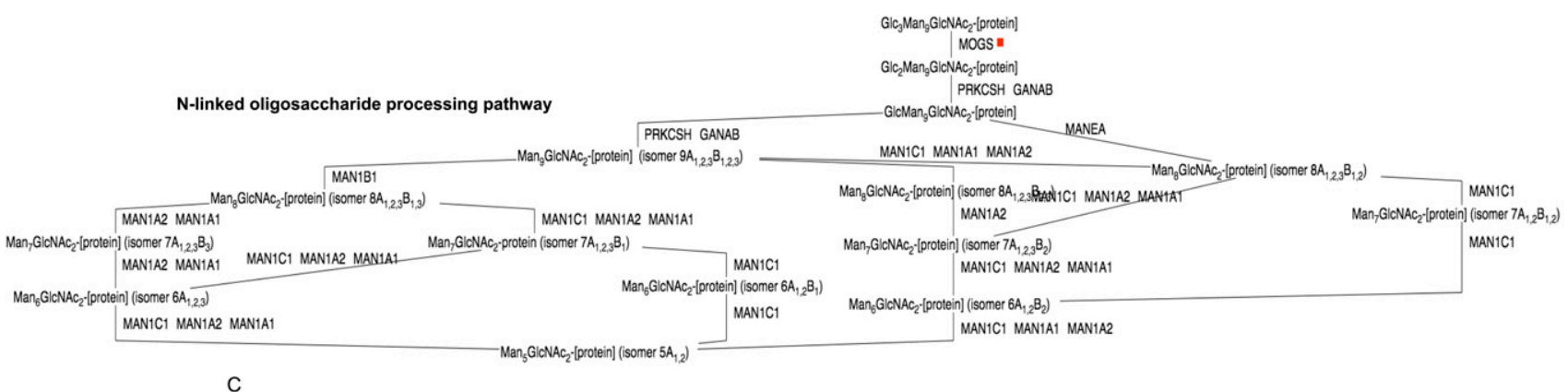

Figure 7. Pi-pathway intersection nodes - Nsp7 and Orf8a in carbohydrate and fatty acid metabolism.

(A) PPi-pathway intersection node-Nsp7. (B) PPi-Pathway intersection node-Orf8a. (C) PPi-pathway intersection node-Nsp7 and Orf8a.

of COVID-19 in the aged population and other severe cases with cytokine storm syndrome.

\section{Immune regulation}

SARS-CoV-2 can proliferate unhindered in infected cells because of the lack of immunity in humans (Felsenstein et al, 2020). The result is cell death, a release of viral particles to the extracellular environment and a general hyperactivity of the immune system in some patients with severe COVID-19 disease and subsequent lung inflammation and cytokine syndrome. Immunocompromised patients or those with underlying symptoms such as diabetes, hypertension, and transplantation are most affected (Zhong et al, 2020). Although clinical trials are ongoing worldwide with various antivirals and immune-modulating treatments, there is currently limited knowledge on the host dependency factors responsible for the individual outcomes of the disease. Our results provide insight into the immune evasion strategies of the SARS-CoV-2 virus; we demonstrate changes in the flux metabolism of vitamin D and tryptophan metabolism during viral infection. Vitamin $\mathrm{D}$ is important for bone growth and turnover and a low vitamin D status is associated with increased susceptibility to upper respiratory tract infections (Mitchell, 2020). Previous studies have shown that supplementation of vitamin D prevents acute respiratory tract infections (Martineau et al, 2017). Our results highlight vitamin D as an essential reaction in the PPi-pathway intersection nodes and we show the viral protein Nsp2 interaction with key enzymes in the vitamins D and C metabolism pathways (Fig 5C). SARS-CoV-2 viral infection causes metabolic perturbations of vitamin D metabolism in the host resulting in disruptions in cellular homeostasis. We propose support therapy management strategies where vitamin D supplements are provided to all COVID-19 patients. Our results also show that tryptophan, melatonin and prostaglandins, important compounds for immunity and homeostasis (Gitto et al, 2011; Platten et al, 2019), are affected by the infection of SARS-CoV-2 and we provide insight into the viral mechanism of action within the human body.

In summary, we have provided a platform for drug target prediction against COVID-19, and future studies on viral entry inhibition, antioxidant therapy, and immune regulation.

\section{Materials and Methods}

For our modelling analyses, we based our reconstruction in the integrated macrophage cell with the SARS-CoV-2 virus model, previously constructed by Renz et al (2020) to develop a new human airway epithelial cell integrated with the SARS-CoV-2 virus. We predicted the reactions which were critical for the survival of the virus in both cell compartments (macrophage and epithelial cells); our results provide insights into COVID-19 treatment management strategies.

\section{GEM reconstruction, curation, and simulations}

We obtained an automated draft reconstruction of the human airway epithelial cell (Wang et al, 2012) and evaluated the metabolic functions and reactions collected in the draft reconstruction against organism-specific literature. We used the gene expression datasets of the human airway epithelial cell (Deprez et al, 2019 Preprint; Vieira Braga et al, 2019) to curate, annotate and improve on the model (Fig 8). We manipulated and simulated the model, including the addition of the gene expression data, with COBRApy (Ebrahim et al, 2013) and GLPK (https://www.gnu.org/software/glpk/). We obtained additional 


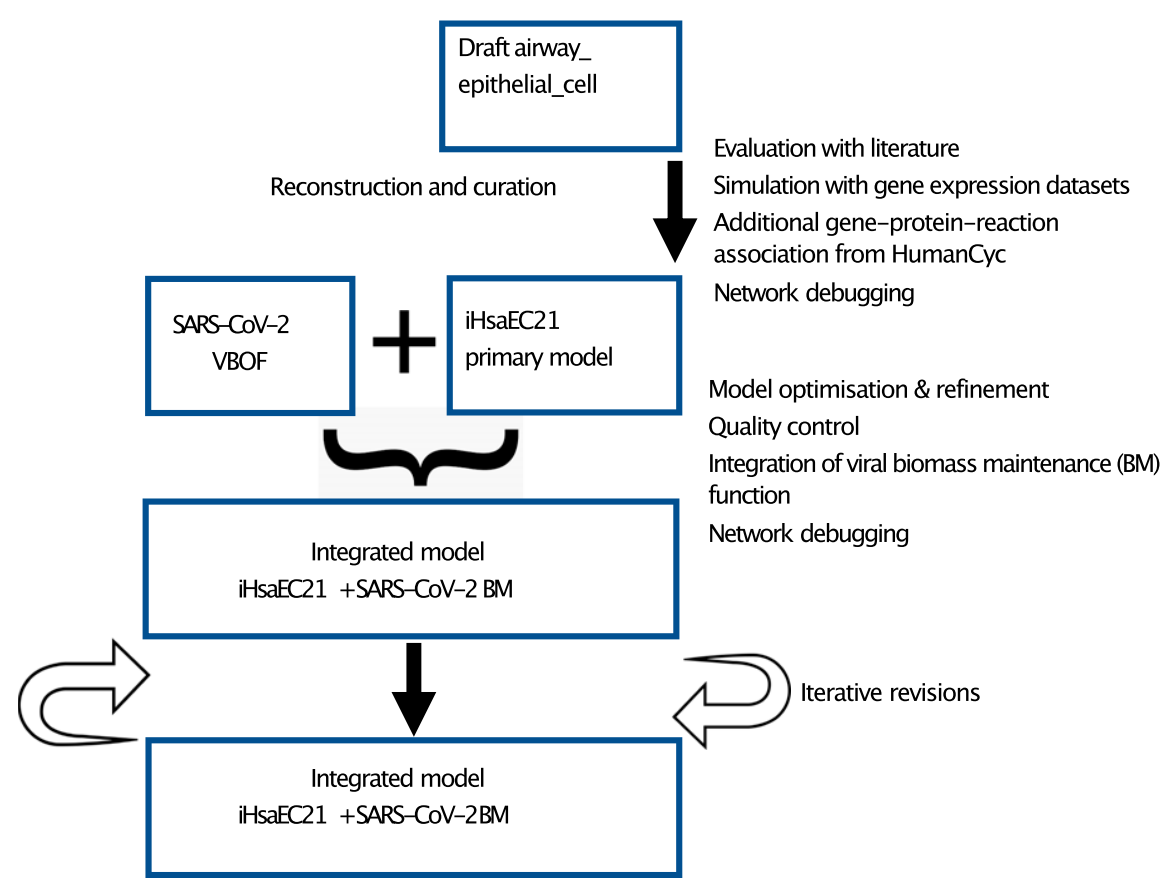

Figure 8. Development of an integrated model of the Human Airway Epithelial cell and SARS-CoV-2.

reactions, gene-to-reaction associations, and pathways (that were not in the automated model) from Humancyc (Trupp et al, 2010) to develop a new genome-scale metabolic model (GEM) of the human airway epithelial cell. We improved on the new reconstruction by mapping the genes and reactions of the GEM to Recon3D, a standard human metabolic model, MetaNetX 4.2, BiGG, ChEBI, MetaCyc, and PubChem databases (Caspi et al, 2014; Hastings et al, 2016; Norsigian et al, 2020; Moretti et al, 2021) and produced a revised human airway epithelial cell iHsaEC21 (Fig 8). The revised model reconstruction, $i$ HsaEC21, can be instantiated without error on the COBRA software (version 0.16.0) (Ebrahim et al, 2013).

\section{Model optimisation}

To improve on the quality of the model, we removed the reactions with dead-end metabolites, which were previously found in the automated model. This was followed by iteratively assessing the model and accounting for the cell-specific metabolic and exchange reactions in the epithelial cell model. We compared and performed various iterations of the new GEM with Recon3D, MetaCyc, MetaNetX 4.2, BiGG, ChEBI, and PubChem databases (Caspi et al, 2014; Hastings et al, 2016; Norsigian et al, 2020; Kim et al, 2021; Moretti et al, 2021). As a result, we added charge and formulae to 1,473 compounds on the new GEM. During the iterations and optimisation of the model, we performed quality control checks on MEMOTE, a standardised genomescale metabolic model testing software (Leiven et al, 2020).

\section{Integration of the human airway epithelial cell model and the SARS-CoV-2 virus}

We integrated the viral biomass maintenance function, previously developed for the macrophage cell (Renz et al. 2020, 2021) into the new GEM to produce an integrated model of iHsaEC21+SARS-CoV-2. We interrogated the new model to identify the host-dependency factors for the SARS-CoV-2 virus by using the novel software tool findCPcli.

The new GEM is encoded in the Systems Biology Markup Language (SBML) (Keating et al, 2020) (iHsaEC21); i for in silico, Hsa for $\mathrm{H}$. sapiens, and EC for airway epithelial cell published in 2021. iHsaEC21 consists of 3,752 reactions, 3,914 metabolites, 4,660 genes, and 48 metabolic pathways.

To assess and predict the performance of the models, we made use of FBA and FVA (Orth et al, 2010). FBA is a computational method that can be applied efficiently to genome-scale models to estimate the fluxes of reactions at a steady state. It is based on the solution of a linear programming problem that maximizes an objective function of interest subject to a set of constraints on the fluxes of the reactions. The linear programming problem associated with FBA can be expressed as follows:

$$
\begin{aligned}
& \max c \cdot v, \\
& \text { s.t. } S \cdot v=0, \\
& L \leq v \leq U,
\end{aligned}
$$

where $v$ is the vector of fluxes, $c$ represents the objective function coefficients, $S$ is the stoichiometry matrix, and $L$ and $U$ are lower and upper bounds on the fluxes. Thus, $c v$ is the objective function, which usually refers to the growth rate of the organism and $S v=0$ represents the balance of fluxes at steady state.

FVA is also based on the solution of linear programming problems, and its main use is the computation of ranges of fluxes that are compatible with given flux constraints. For instance, if the growth rate predicted by FBA is $\mu_{\max }$, then the range of fluxes of a given reaction $i$ that are compatible with such growth rate can be 
obtained by minimizing and maximizing the following programming problem:

$$
\begin{gathered}
\min / \max v_{i}, \\
\text { s.t. } S \cdot v=0, \\
L \leq v \leq U, \\
v_{\text {growth }}=\mu_{\max },
\end{gathered}
$$

where $v_{\text {growth }}$ is the flux of the reaction associated with growth and $v_{i}$ is the flux of reaction $i$. FBA and FVA were computed on the metabolic network of the host, both with and without the reaction modelling the production of the virus, by using the Python toolbox COBRApy.

Bottleneck reactions, like chokepoint reactions, are required for the reaction synthesis and the removal of these reactions will cause an accumulation or depletion of the metabolites; thus, they represent potential drug targets. The software tool findCPcli was developed to compute bottleneck reactions on genome-scale models by considering the structural and dynamic information of the models. The dynamic information is considered as follows: (a) FVA is run to compute lower and upper flux bounds of the reactions; (b) the obtained flux bounds are used to identify reversible and nonreversible; and (c) this directionality of reactions is used to determine consumer and producer reactions, and in turn, bottleneck reactions. In addition to the computation of bottleneck reactions, findCPcli can also compute and remove dead-end metabolites, find essential reactions, and update the flux bounds of the reactions according to the results of FVA.

\section{Data Availability}

The model has been deposited as an SBML Level 3 Version 1 (Hucka et al, 2018) file with FBC extension and the minimal information required in the annotation of models (MIRIAM) (Juty et al, 2012) in BioModels (https:// www.ebi.ac.uk/biomodels/MODEL2007210001) (Malik-Sheriff et al, 2020). The source code of findCPcli together with its documentation is available at https://github.com/findCP/findCPcli. The tool requires Python 3.5 (or higher) and can be installed with pip, the standard package installer for Python. The pathway maps were created with the pathway collage software (Paley et al, 2016) and the essential reactions are available as a smart table on HumanCyc (https:// biocyc.org/group?id=biocyc17-293513833343490).

\section{Supplementary Information}

Supplementary Information is available at https://doi.org/10.26508/lsa. 202000954.

\section{Acknowledgements}

The authors of this manuscript are supported by The Wellcome Trust 107032AIA (RA Floto, BP Bannerman) The UK Cystic Fibrosis Trust (Innovation
Hub grant 001) (RA Floto), and the Spanish Ministry of Science, Innovation, and Universities (J Júlvez and A Oarga). The authors would like to thank Jo Chukualim for the graphics and illustrations. The authors would also like to thank Charlotte Passemar for slides with preliminary data demonstrating the expression of ACE2 in epithelial cells.

\section{Author Contributions}

BP Bannerman: conceptualization, data curation, software, formal analysis, investigation, methodology, and writing-original draft, review, and editing.

J Júlvez: data curation, software, formal analysis, investigation, and writing-original draft, review, and editing.

A Oarga: data curation, software, formal analysis, and writingoriginal draft, review, and editing.

TL Blundell: formal analysis and writing-review and editing.

P Moreno: data curation, formal analysis, investigation, and writingreview and editing.

RA Floto: formal analysis, investigation, and writing-review and editing.

\section{Conflict of Interest Statement}

The authors declare that they have no conflict of interest.

\section{References}

Abu-Farha M, Thanaraj TA, Qaddoumi MG, Hashem A, Abubaker J, Al-Mulla F (2020) The role of lipid metabolism in COVID-19 virus infection and as a drug target. Int J Mol Sci 21: 3544. doi:10.3390/ijms21103544

Aller S, Scott A, Sarkar-Tyson M, Soyer OS (2018) Integrated human-virus metabolic stoichiometric modelling predicts host-based antiviral targets against Chikungunya, Dengue and Zika viruses. J R Soc Interf 15: 20180125. doi:10.1098/rsif.2018.0125

Blaising J, Lévy PL, Polyak SJ, Stanifer M, Boulant S, Pécheur El (2013) Arbidol inhibits viral entry by interfering with clathrin-dependent trafficking. Antiviral Res 100: 215-219. doi:10.1016/j.antiviral.2013.08.008

Bordbar A, Lewis NE, Schellenberger J, Palsson B $\varnothing$, Jamshidi N (2010) Insight into human alveolar macrophage and M. tuberculosis interactions via metabolic reconstructions. Mol Syst Biol 6: 422. doi:10.1038/ msb.2010.68

Brunk E, Sahoo S, Zielinski DC, Altunkaya A, Dräger A, Mih N, Gatto F, Nilsson A Preciat Gonzalez GA, Aurich MK, et al (2018) Recon3D enables a threedimensional view of gene variation in human metabolism. Nat Biotechnol 36: 272-281. doi:10.1038/nbt.4072

Carey MA, Dräger A, Beber ME, Papin JA, Yurkovich JT (2020) Community standards to facilitate development and address challenges in metabolic modeling. Mol Syst Biol 16: e9235. doi:10.15252/ msb.20199235

Caspi R, Altman T, Billington R, Dreher K, Foerster H, Fulcher CA, Holland TA, Keseler IM, Kothari A, Kubo A, et al (2014) The MetaCyc database of metabolic pathways and enzymes and the BioCyc collection of pathway/genome databases. Nucleic Acids Res 42: D459-D471. doi:10.1093/nar/gkt1103

Chen Y, Liu Q, Guo D (2020) Emerging coronaviruses: Genome structure, replication, and pathogenesis. J Med Virol 92: 418-423. doi:10.1002/ jmv.25681

Danis K, Epaulard O, Bénet T, Gaymard A, Campoy S, Botelho-Nevers E, Bouscambert-Duchamp M, Spaccaferri G, Ader F, Mailles A, et al (2020) 
Cluster of coronavirus disease 2019 (COVID-19) in the French alps, February 2020. Clin Infect Dis 71: 825-832. doi:10.1093/cid/ciaa424

Delgado-Roche L, Mesta F (2020) Oxidative stress as key player in severe acute respiratory syndrome coronavirus (SARS-CoV) infection. Arch Med Res 51: 384-387. doi:10.1016/j.arcmed.2020.04.019

Deprez M, Zaragosi L, Truchi M, Garcia SR, Arguel MJ, Lebrigand K, Paquet A, Pee'r D, Marquette C-H, Leroy S, et al (2019) A single-cell atlas of the human healthy airways. BioRxiv doi:10.1101/2019.12.21.884759 (Preprint posted December 23, 2019).

Devaux CA, Rolain J-M, Colson P, Raoult D (2020) New insights on the antiviral effects of chloroquine against coronavirus: What to expect for COVID19? Int J Antimicrobial Agents 55: 105938. doi:10.1016/ j.ijantimicag.2020.105938

de Wilde AH, Jochmans D, Posthuma CC, Zevenhoven-Dobbe JC, van Nieuwkoop S, Bestebroer TM, van den Hoogen BG, Neyts J, Snijder EJ (2014) Screening of an FDA-approved compound library identifies four small-molecule inhibitors of Middle East respiratory syndrome coronavirus replication in cell culture. Antimicrob Agents Chemother 58: 4875-4884. doi:10.1128/AAC.03011-14

Dimitrov DS (2004) Virus entry: Molecular mechanisms and biomedical applications. Nat Rev Microbiol 2: 109-122. doi:10.1038/nrmicro817

Donoghue M, Hsieh F, Baronas E, Godbout K, Robinson K, Jeyaseelan R, Breitbart RE, Acton S (2000) A novel angiotensin-converting enzyme-related carboxypeptidase (ACE2) converts angiotensin I to angiotensin 1-9. Clin Res 87: e1-e9. doi:10.1161/01.res.87.5.e1

Duarte NC, Becker SA, Jamshidi N, Thiele I, Mo ML, Vo TD, Srivas R, Palsson B $\varnothing$ (2007) Global reconstruction of the human metabolic network based on genomic and bibliomic data. Proc Natl Acad Sci U S A 104: 1777-1782. doi:10.1073/pnas.0610772104

Dyer O (2020) Covid-19: Remdesivir has little or no impact on survival, WHO trial shows. BMJ 371: m4057. doi:10.1136/bmj.m4057

Ebrahim A, Lerman JA, Palsson BO, Hyduke DR (2013) COBRApy: Constraintsbased reconstruction and analysis for Python. BMC Syst Biol 7: 74. doi:10.1186/1752-0509-7-74

Felsenstein S, Herbert JA, McNamara PS, Hedrich CM (2020) COVID-19: Immunology and treatment options. Clin Immunol 215: 108448. doi:10.1016/j.clim.2020.108448

Fiévet C, Staels B (2009) Combination therapy of statins and fibrates in the management of cardiovascular risk. Curr Opin Lipidol 20: 505-511. doi:10.1097/mol.0b013e328332e9ef

Foyer CH, Noctor G (2005) Redox homeostasis and antioxidant signaling: A metabolic interface between stress perception and physiological responses. Plant Cell 17: 1866-1875. doi:10.1105/tpc.105.033589

Gitto E, Aversa S, Reiter RJ, Barberi I, Pellegrino S (2011) Update on the use of melatonin in pediatrics. J Pineal Res 50: 21-28. doi:10.1111/j.1600079X.2010.00814.X

Gordon DE, Jang GM, Bouhaddou M, Xu J, Obernier K, White KM, O'Meara MJ, Rezelj VV, Guo JZ, Swaney DL, et al (2020) A SARS-CoV-2 protein interaction map reveals targets for drug repurposing. Nature 583 459-468. doi:10.1038/s41586-020-2286-9

Hamming I, Timens W, Bulthuis ML, Lely AT, Navis G, van Goor H (2004) Tissue distribution of ACE2 protein, the functional receptor for SARS coronavirus. A first step in understanding SARS pathogenesis. J Pathol 203: 631-637. doi:10.1002/ path.1570

Hastings J, Owen G, Dekker A, Ennis M, Kale N, Muthukrishnan V, Turner S, Swainston N, Mendes P, Steinbeck C (2016) ChEBI in 2016: Improved services and an expanding collection of metabolites. Nucleic Acids Res 44: D1214-D1219. doi:10.1093/nar/gkv1031

Heaton NS, Randall G (2011) Multifaceted roles for lipids in viral infection. Trends Microbiol 19: 368-375. doi:10.1016/j.tim.2011.03.007

Hoppe HC, van Schalkwyk DA, Wiehart UI, Meredith SA, Egan J, Weber BW (2004) Antimalarial quinolines and artemisinin inhibit endocytosis in
Plasmodium falciparum. Antimicrob Agents Chemother 48: 2370-2378. doi:10.1128/AAC.48.7.2370-2378.2004

Horowitz RI, Freeman PR, Bruzzese J (2020) Efficacy of glutathione therapy in relieving dyspnea associated with COVID-19 pneumonia: A report of 2 cases. Respir Med Case Rep 30: 101063. doi:10.1016/j.rmcr.2020.101063

Huang C, Wang Y, Li X, Ren L, Zhao J, Hu Y, Zhang L, Fan G, Xu J, Gu X, et al (2020) Clinical features of patients infected with 2019 novel coronavirus in Wuhan, China. Lancet 395: 497-506. doi:10.1016/S0140-6736(20)30183-5

Hucka M, Bergmann FT, Dräger A, Hoops S, Keating SM, Le Novère N, Myers C), Olivier BG, Sahle S, Schaff JC, et al (2018) The systems biology markup Language (SBML): Language specification for level 3 version 2 core. J Integr Bioinform 15: 20170081. doi:10.1515/jib-2017-0081

Juty N, Le Novère N, Laibe C (2012) Identifiers.org and MIRIAM registry: Community resources to provide persistent identification. Nucleic Acids Res 40: D580-D586. doi:10.1093/nar/gkr1097

Kanehisa M, Furumichi M, Sato Y, Ishiguro-Watanabe M, Tanabe M (2021) KEGG: Integrating viruses and cellular organisms. Nucleic Acids Res 49: D545-D551. doi:10.1093/nar/gkaa970

Keating SM, Waltemath D, König M, Zhang F, Dräger A, Chaouiya C, Bergmann FT, Finney A, Gillespie CS, Helikar T, et al (2020) SBML level 3: An extensible format for the exchange and reuse of biological models. Mol Syst Biol 16: e9110. doi:10.15252/msb.20199110

Kim S, Chen J, Cheng T, Gindulyte A, He J, He S, Li Q, Shoemaker BA, Thiessen PA, Yu B, et al (2021) PubChem in 2021: New data content and improved web interfaces. Nucleic Acids Res 49: D1388-D1395. doi:10.1093/nar/ gkaa971

Norsigian CJ, Pusarla N, McConn JL, Yurkovich JT, Dräger A, Palsson BO, King Z (2020) BiGG models 2020: Multi-strain genome-scale models and expansion across the phylogenetic tree. Nucleic Acids Res 48 D402-D406. doi:10.1093/nar/gkz1054

Li R, Pei S, Chen B, Song Y, Zhang T, Yang W, Shaman J (2020) Substantial undocumented infection facilitates the rapid dissemination of novel coronavirus (SARS-CoV2). Science 368: 489-493. doi:10.1126/ science.abb3221

Lieven C, Beber ME, Olivier BG, Bergmann FT, Ataman M, Babaei P, Bartell JA Blank LM, Chauhan S, Correia K, et al (2020) MEMOTE for standardized genome-scale metabolic model testing. Nat Biotechnol 38: 272-276. doi:10.1038/s41587-020-0446-y

Liu Y, Qu HQ, Qu J, Tian L, Hakonarson H (2020) Expression pattern of the SARS-CoV-2 entry genes ACE2 and TMPRSS2 in the respiratory tract. Viruses 12: 1174. doi:10.3390/v12101174

Malik-Sheriff RS, Glont M, Nguyen TVN, Tiwari K, Roberts MG, Xavier A, Vu MT, Men J, Maire M, Kananathan S, et al (2020) BioModels-15 years of sharing computational models in life science. Nucleic Acids Res 48: D407-D415. doi:10.1093/nar/gkz1055

Martineau AR, Jolliffe DA, Hooper RL, Greenberg L, Aloia JF, Bergman P, Dubnov-Raz G, Esposito S, Ganmaa D, Ginde AA, et al (2017) Vitamin D supplementation to prevent acute respiratory tract infections: Systematic review and meta-analysis of individual participant data. BMJ 356: i6583. doi:10.1136/bmj.i6583

Mazzon M, Marsh M (2019) Targeting viral entry as a strategy for broadspectrum antivirals. F1000Res 8: 1628. doi:10.12688/f1000research. 19694.1

Mitchell F (2020) Vitamin-D and COVID-19: Do deficient risk a poorer outcome? Lancet Diabetes Endocrinol 8: 570. doi:10.1016/S22138587(20)30183-2

Moretti S, Tran VDT, Mehl F, Ibberson M, Pagni M (2021) MetaNetX/MNXref: Unified namespace for metabolites and biochemical reactions in the context of metabolic models. Nucleic Acids Res 49: D570-D574. doi:10.1093/nar/gkaa992

Müller C, Hardt M, Schwudke D, Neuman BW, Pleschka S, Ziebuhr J (2018) Inhibition of cytosolic phospholipase A2 $\alpha$ impairs an early step of 
coronavirus replication in cell culture. J Virol 92: e01463-17. doi:10.1128/JVI.01463-17

Munro R, Morrison E, McDonald AG, Hunter JA, Madhok R, Capell HA (1997) Effect of disease modifying agents on the lipid profiles of patients with rheumatoid arthritis. Ann Rheum Dis 56: 374-377. doi:10.1136/ $\operatorname{ard} .56 .6 .374$

Oarga A, Bannerman B, Júlvez J (2020) Lecture notes in computer science. Growth dependent computation of chokepoints in metabolic networks. In Computational Methods in Systems Biology, Vol. 12314 Cham, Switzerland: Springer.

Orth JD, Thiele I, Palsson $B \varnothing$ (2010) What is flux balance analysis? Nat Biotechnol 28: 245-248. doi:10.1038/nbt.1614

Ostaszewski M, Mazein A, Gillespie ME, Kuperstein I, Niarakis A, Hermjakob H, Pico AR, Willighagen EL, Evelo CT, Hasenauer J, et al (2020) COVID-19 Disease Map, building a computational repository of SARS-CoV-2 virus-host interaction mechanisms. Sci Data 7: 136. doi:10.1038/ s41597-020-0477-8

Paley S, O'Maille PE, Weaver D, Karp PD (2016) Pathway collages: Personalized multi-pathway diagrams. BMC Bioinformatics 17: 529. doi:10.1186/ s12859-016-1382-1

Pan H, Pan H, Peto R, Henao-Restrepo AM, Preziosi MP, Sathiyamoorthy V, Abdool Karim Q, Alejandria MM, Hernández García C, Kieny MP, et al (2021) Repurposed antiviral drugs for covid-19: Interim WHO solidarity trial results. N Engl J Med 384: 497-511. doi:10.1056/NEJMoa2023184

Platten M, Nollen EAA, Röhrig UF, Fallarino F, Opitz CA (2019) Tryptophan metabolism as a common therapeutic target in cancer, neurodegeneration and beyond. Nat Rev Drug Discov 18: 379-401. doi:10.1038/s41573-019-0016-5

Renz A, Widerspick L, Dräger A (2020) FBA reveals guanylate kinase as a potential target for antiviral therapies against SARS-CoV-2. Bioinformatics 36: i813. doi:10.1093/bioinformatics/btaa813

Renz A, Widerspick L, Dräger A (2021) Genome-scale metabolic model of infection with SARS-CoV-2 mutants confirms guanylate kinase as robust potential antiviral target. Genes (Basel) 12: 796. doi:10.3390/ genes12060796

Seaver SMD, Liu F, Zhang Q, Jeffryes J, Faria JP, Edirisinghe JN, Mundy M, Chia N, Noor E, Beber ME, et al (2021) The ModelSEED Biochemistry Database for the integration of metabolic annotations and the reconstruction, comparison and analysis of metabolic models for plants, fungi and microbes. Nucleic Acids Res 49: D575-D588. doi:10.1093/nar/gkaa1143

Steward J (2020) Host pathways in coronavirus replication and COVID-19 pre-clinical drug target identification using proteomic and chemoinformatic analysis. Drug Target Rev. https:/ / www.drugtargetreview.com/article/58628/ host-pathwaysin-coronavirus-replication-and-covid-19-pre-clinical-drug-targetidentification-using-proteomic-and-chemoinformatic-analysis/

Trupp M, Altman T, Fulcher CA, Caspi R, Krummenacker M, Paley S, Karp PD (2010) Beyond the genome (BTG) is a (PGDB) pathway genome database: HumanCyc. Genome Biol 11: 012. doi:10.1186/gb-2010-11s1-012

Uzun T, Toptas O (2020) Artesunate: Could be an alternative drug to chloroquine in COVID-19 treatment? Chin Med 15: 54. doi:10.1186/ s13020-020-00336-8

Vieira Braga FA, Kar G, Berg M, Carpaij OA, Polanski K, Simon LM, Brouwer S Gomes T, Hesse L, Jiang J, et al (2019) A cellular census of human lungs identifies novel cell states in health and in asthma. Nat Med 25 1153-1163. doi:10.1038/s41591-019-0468-5

Wang H, Yang P, Liu K, Guo F, Zhang Y, Zhang G, Jiang C (2008) SARS coronavirus entry into host cells through a novel clathrin- and caveolae-independent endocytic pathway. Cell Res 18: 290-301. doi:10.1038/cr.2008.15

Wang Y, Eddy JA, Price ND (2012) Reconstruction of genome-scale metabolic models for 126 human tissues using mCADRE. BMC Syst Biol 6: 153. doi:10.1186/1752-0509-6-153

Wishart DS, Feunang YD, Marcu A, Guo AC, Liang K, Vázquez-Fresno R, Sajed T, Johnson D, Li C, Karu N, et al (2018) HMDB 4.0: The human metabolome database for 2018. Nucleic Acids Res 46: D608-D617. doi:10.1093/nar/ gkx1089

Yeh I, Hanekamp T, Tsoka S, Karp PD, Altman RB (2004) Computational analysis of plasmodium falciparum metabolism: Organizing genomic information to facilitate drug discovery. Genome Res 14: 917-924. doi:10.1101/gr.2050304

Zhong J, Tang J, Ye C, Dong L (2020) The immunology of COVID-19: Is immune modulation an option for treatment? Lancet Rheumatol 2: e428-e436. doi:10.1016/S2665-9913(20)30120-X

Zhou P, Yang XL, Wang XG, Hu B, Zhang L, Zhang W, Si HR, Zhu Y, Li B, Huang CL, et al (2020) A pneumonia outbreak associated with a new coronavirus of probable bat origin. Nature 579: 270-273. doi:10.1038/s41586-0202012-7

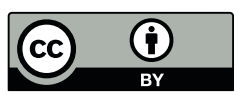

License: This article is available under a Creative Commons License (Attribution 4.0 International, as described at https://creativecommons.org/ licenses/by/4.0/). 